\title{
Bias Adjustment Techniques Are Underutilized in HIV Sexual Risk Estimation: A Systematic Review
}

\author{
Nguyen K. Tran, Neal D. Goldstein and Seth L. Welles * \\ Department of Epidemiology and Biostatistics, Dornsife School of Public Health, Drexel University, Nesbitt Hall, \\ 3215 Market St., Philadelphia, PA 19104, USA; nt448@drexel.edu (N.K.T.); ng338@drexel.edu (N.D.G.) \\ * Correspondence: slw58@drexel.edu; Tel.: +1-267-359-6203
}

Received: 8 March 2018; Accepted: 7 August 2018; Published: 9 August 2018

\begin{abstract}
Background: Valid measurement of determinants of HIV infection among men who have sex with men (MSM) is critical for intervention planning and resource allocation. However, sexual minority research concerning HIV risk often relies on proxy exposures of sexual behaviors such as sexual orientation and partner gender. Inferring high risk sexual behaviors (i.e., condomless anal intercourse) from these proxies inaccurately captures HIV risk, but few studies have attempted to correct for this bias. Methods: We performed a systematic review of methodological practices for estimating risk of HIV infection among MSM. Results: We identified 32 studies in which high risk sexual behavior was assessed: $82 \%(n=26)$ measured and used sexual risk behaviors (e.g., condomless anal intercourse or sexual positioning) to assess risk of HIV infection; $9 \%(n=3)$ used proxy measures; and $9 \%(n=3)$ used both behavior and proxy variables. Various treatments of misclassification reported by investigators included the following: $82 \%(n=26)$ discussed misclassification of sexual behavior as a potential limitation; however, among these studies, no attempts were made to correct misclassification; $12 \%(n=4)$ did not report exposure misclassification, and 6\% $(n=2)$ explicitly considered this information bias and conducted a Bayesian approach to correct for misclassification. Conclusions: Our systematic review indicates that a majority of studies engaging in collecting primary data have taken additional steps to acquire detailed information regarding sexual risk behaviors. However, reliance on population-based surveys may still lead to potentially biased estimates. Thus, bias analytic techniques are potential tools to control for any suspected biases.
\end{abstract}

Keywords: HIV/AIDS; men who have sex with men; misclassification; exposure assessment

\section{Introduction}

Human sexuality is a complex construct, which involves considering three key aspects: sexual orientation (how individuals describe or identify their sexuality), sexual attraction (to whom an individual is sexually attracted regardless of biological sex or gender identity), and sexual behaviors (with whom an individual says they have sexual experiences) [1,2]. When evaluating HIV risk among gay and bisexual men, it is the actual sexual behavior (i.e., condomless anal sex) that transmits the pathogen and not how the individual identifies his or her sexuality [3].

Unfortunately, many generalizable surveys that attempt to capture population health at a broad level, such as the National Health and Nutrition Examination Survey (NHANES), collect data on sexual behaviors in a limited and problematic manner. Rather, they rely upon proxy measures of behavior, such as sexual orientation. The use of proxies may be intentional since these surveys need to balance breadth versus depth. Nevertheless, the data captured are not ideal for examining HIV transmission, since some men who identify as gay or bisexual may engage in low risk same-sex behaviors (e.g., oral sex). Further complicating the issue, surveys that collect socially sensitive data on potentially stigmatizing factors such as same-sex orientation and behavior are prone to 
underreporting $[4,5]$, potentially compromising the important public health charge of accurately measuring HIV risk in high risk communities.

One possible scenario is that people identify as heterosexual or straight, but also engage in sex with partners of the same gender [6]. In epidemiology, this phenomenon is known as misclassification, which threatens internal validity of study findings. It is important to note that this is not a misclassification of their orientation (being gay/homosexual or bisexual), but inferring sexual behaviors from self-reported sexual orientation can be problematic when assessing HIV risk. Any error introduced in exposure assessment is carried forward into the analytic modeling of the outcome and possibly rendering the predictions unreliable.

\section{Consequences of Misclassifying Sexual Behavior in HIV Risk Estimation}

Men that identify as gay, bisexual, or have sex with men (MSM) are overrepresented among new HIV cases in the United States [3]. As described, MSM behavior may be denoted by self-reported sexual orientation, same-sex attraction, sexual relationships with other men (same-sex partner), or a combination of these identifying factors. MSM from communities where same-sex behaviors and identities are stigmatized tend to conceal such information, resulting in misclassification of their potential risk for HIV transmission [7,8]. Further, this misclassification may be differential, in that knowledge of one's HIV serostatus may affect how they report sexuality. Prior work has shown that individuals with and without a history of sexually transmitted infections have different probabilities of reporting gay identity or same-sex behaviors $[7,8]$. Accordingly, this differential misclassification may bias HIV risk estimates among gay and bisexual men. Research that relies on partner gender for assessing risk of transmitting or acquiring HIV is likely to be less biased than research that relies on reported sexual identity [9]. However, even partner gender may not capture risk entirely accurately; for example, an individual may have male sex partners but engage entirely in oral sex, conferring a lower risk for infection.

Among transgender individuals, assessing this high-risk behavior through proxy variables is further complicated by the range of genital configurations; transgender individuals may or may not have same-sex attraction or identity regardless of their genitalia. Thus, rather than rely on sexual and/or gender identity, or partner gender, it is crucial to identify individuals who engage in receptive anal intercourse, which confers the greatest risk for infection.

Without optimal data, researchers must rely upon bias correction methodology [10], but it is currently unclear how often these techniques are used in HIV and other sexually transmitted infections research to obtain less biased estimates of risk in sexual minority groups. In addition, Pathela et al. [6] and Igartua et al. [11] have heeded the need to improve exposure assessment of high risk sexual behaviors. This sentiment is relatively prevalent as several studies (approximately a total of 200 cited articles) have referenced these articles. Therefore, in this paper, we sought to examine how often assessment of risk of HIV transmission due to condomless anal intercourse is evaluated via commonly reported proxy variables (such as self-reported sexual orientation or gender of partners), further highlighting the need to adopt recent methodology to adjust for this misclassification.

\section{Methods}

We conducted a systematic review of the literature, targeting peer-reviewed publications that have reported methods for estimating risk of HIV infection among MSM. This systematic review followed the Preferred Reporting Items for Systematic Reviews and Meta-Analyses (PRISMA) guidelines [12] and previous approaches in cataloging methods in high-impact literature $[13,14]$. Our goals were to (1) document reports of potential misclassification of high risk sexual behaviors in observational studies focusing on sexual minority men and HIV transmission, and (2) characterize the use of bias adjustment methods in correcting the proxies for sexual risk behaviors. 
We selected 14 widely read journals publishing in areas of HIV infection and diseases, clinical research, and epidemiological methods and searched for potential studies assessing the risk of HIV acquisition using proxy variables to capture condomless anal intercourse and categorizing men based on these proxy measures. These journals included:

- HIV- and STI-focused journals: Acquired Immunodeficiency Syndrome, Journal of Acquired Immunodeficiency Syndrome, Acquired Immunodeficiency Syndrome and Behavior, Sexually Transmitted Disease, Sexually Transmitted Infections,

- Epidemiology-focused journals: American Journal of Epidemiology, Epidemiology, International Journal of Epidemiology, European Journal of Epidemiology, and Annals of Epidemiology,

- General medical journals: New England Journal of Medicine, Journal of American Medical Association, Lancet, and Annals of Internal Medicine.

We searched through keywords, titles, and abstracts using the following terms (Figure 1): (Men who have sex with men OR MSM OR gay OR homosexual OR bisexual OR same-sex OR high-risk sexual behavior) AND (human immunodeficiency virus OR HIV). To avoid limiting our search results, particularly in epidemiological and medical journals that may have fewer publications among sexual minority men and HIV infection, we broadened the scope of our search and used only the terms men who have sex with men OR HIV.

\section{Study Inclusion/Exclusion Criteria}

Articles were eligible for inclusion in our study if (1) the publication date was between January 2010 to December 2017; (2) study design was observational; (3) there was a sample/subsample of sexual minority men (i.e., gay, bisexual, or men who have sex with men); (4) high risk sexual behaviors or proxies of such behaviors were evaluated as an exposure of interest; and (5) assessment of HIV infection among sexual minority men as one of the primary outcomes. We excluded studies prior to 2010 to present a description of the current methodology (or lack thereof) in operationalizing high-risk sexual behaviors and potential methods of adjustment when such proxies (e.g., sexual identity) are used. Simulation modeling studies, randomized controlled trials (RCT), and case studies were excluded from our review as these study designs are not subjected to misclassification bias in a similar manner as observation studies: (1) modeling studies draw from previous estimates; therefore, if the estimates itself were not biased then assumptions for models relatively accurate; (2) RCT are less prone to bias as there is greater control exerted by the investigator to accurately measure the primary exposure of interest; and (3) case studies strictly limits itself to pure description; therefore, discussion of inferences does not extend beyond the observations. Also excluded were brief reports, qualitative studies, and studies that did not analyze individual level factors. In addition, we focused on cisgender MSM as they possess the greater burden of HIV infection.

We defined proxies for high risk sexual behaviors as sexual identity, same-sex partner, or same-sex attraction. Same-sex partnerships were classified as ever having sex with a male partner or within the last 12 months. Results were extracted and presented for the following information from each article: (1) how the questionnaire was administered to participants; (2) methods in capturing high risk sexual behaviors such as condomless anal sex; (3) when measurement of such behavior was absent, how MSM was operationalized as the exposure using self-reported proxies for high-risk sexual behaviors such as sexual orientation or gender of sexual partners; (4) acknowledgement of potential misclassification due to inaccurate methodology; and (5) adjustment for purported misclassification of sexual behavior proxy. 


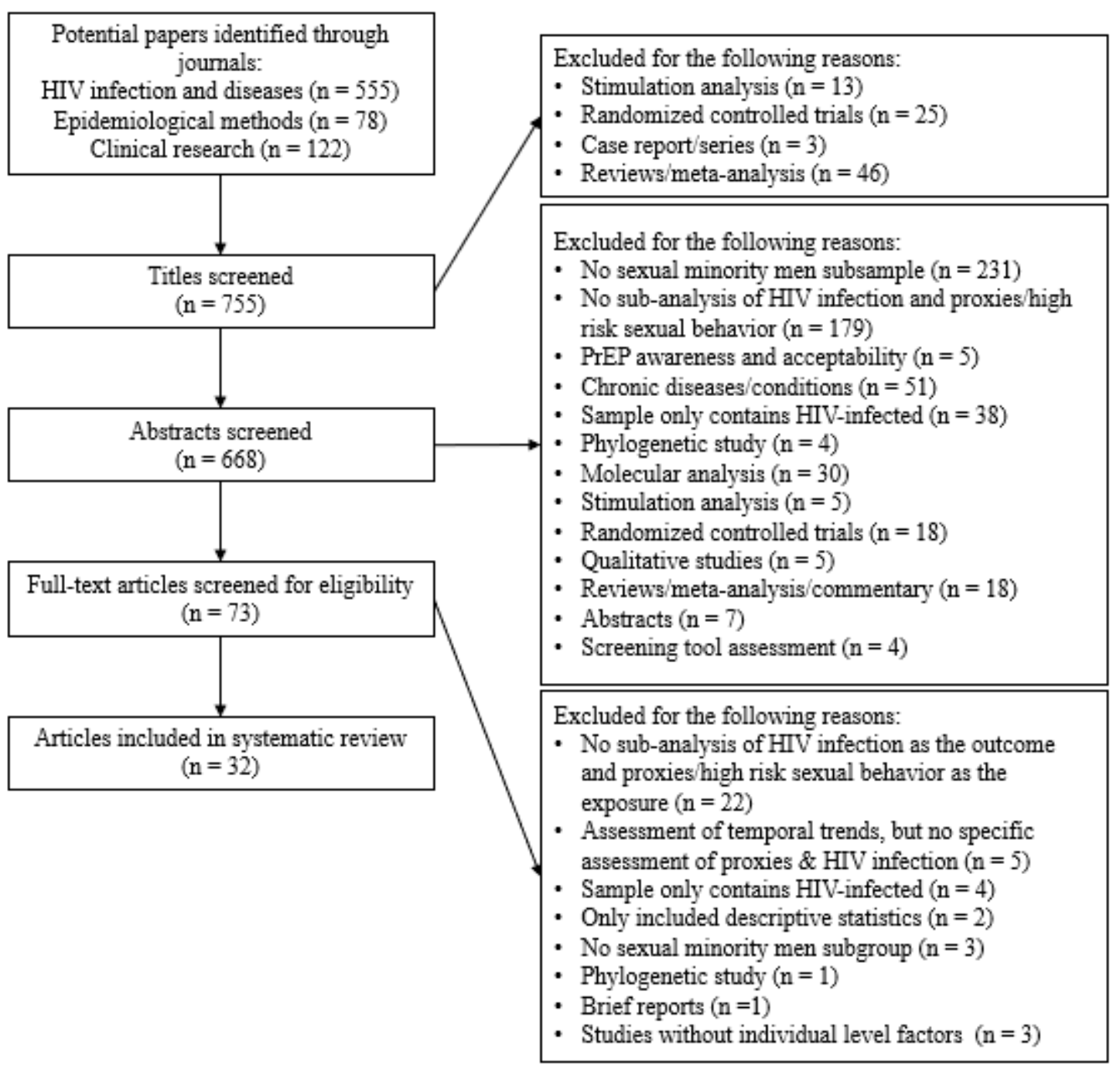

Figure 1. PRISMA Flow Chart of Systematic Search.

\section{Results}

\subsection{Assessment of High-Risk Behaviors and MSM Exposure}

The search criteria identified 755 potentially relevant manuscripts. Of these potential papers, $87(12 \%)$ articles were excluded because the title included simulation analysis, RCT, case reports/series, or other systematic reviews/meta-analyses. Among the remaining 668 articles, we further excluded 595 (89\%) articles primarily for one of the following reasons: a subsample of sexual minority men was not detected in the study $(n=231)$ or the assessment did not specifically include a sub-analysis evaluating the association of HIV infection with high risk sexual behaviors or proxies $(n=179)$. A total of $73(10 \%)$ articles were retained for full-text examination. Of these, 41 studies (56\%) were further removed as they did not have a sub-analysis focusing on sexual risk exposure and HIV infection. Our final sample was $32(44 \%)$ published manuscripts. Studies in this systematic review included diverse MSM populations, including international studies in Asia [15-20], Africa [21-23], South America [24,25], and Europe [26,27] (41\%); Black and Latino persons in the United States [28-35] (25\%); and young adults or adolescents [36-39] (13\%). Half of studies (50\%) used only community-based surveys $[15,16,18,20,21,24,25,28,29,32,35,37,38,40-42], 38 \%(n=12)$ used population-based surveys [17,19,22,23,26,33,34,36,39,43-45], 9\% $(n=3)$ used both community and population-based surveys [27,30,31], and one study $(3 \%)$ used surveillance data to conduct their 
analysis [46]. All studies were quantitative in nature and assessed the association between HIV infection and various demographic and behavioral characteristics among MSM.

\subsection{Self-Reported Proxy of High-Risk Sexual Behavior}

Review of each study indicated that self-reported proxy of high-risk sexual behavior used to classify MSM generally fell into one of two categories: sexual identity or partner gender (Table 1). Studies generally used dichotomous (yes/no) questions to ascertain sexual identity and partner gender. These proxies were assessed using self-identified sexual orientation (i.e., heterosexual, gay/homosexual, bisexual, or not sure) or same-sex partners during the previous 12 months or lifetime. In total, $9 \%(n=3)[34,43,46]$ used proxies exclusively (i.e., sexual identity or partner gender), $82 \%(n=26)[15-23,25-29,32,33,35-42,44,45]$ used sexual risk behaviors and did not rely on proxy measures, and $9 \%(n=3)[24,30,31]$ used a combination of these measures to evaluate HIV transmission among MSM.

\subsection{Acknowledgement of Purported Sexual Behavior Misclassification}

Among the 32 studies assessing the association of HIV infection with high risk sexual behaviors or proxy variables, $12 \%(n=4)[28,33,38,39]$ did not report any potential misclassification and $82 \%(n=26)[15-27,29,32,34-37,40-46]$ only discussed misclassification of sexual behavior or MSM classification as a potential limitation. In one such study, clearly stated by Reisner et al. [34], "There was no assessment of recent HIV sexual risk behavior (e.g., unprotected vaginal or anal intercourse) in the NESARC data; therefore, we could not describe or consider recent behavioral risk patterns that may have led to incident HIV infections in our study." Similar approaches in reporting potential misclassification bias was observed in other articles; however, among these studies, statistical techniques to adjust for misclassification were not employed.

Two studies (6\%) explicitly considered this information bias [30,31]. In the work by Goldstein et al. [31] that examined data from NESARC, the authors noted that MSM had nearly 21 times the odds [95\% confidence interval (CI): 6.3, 61.1] of HIV infection compared to non-MSM when classifying higher-risk sexual behavior using self-reported sexual identity. After correcting for potential misclassification of behavior, the authors note this risk estimate was artificially inflated (as men may engage in sex with other men, but not identify as gay/homosexual), and that a more plausible (yet still greatly increased) estimate was approximately a five-fold increase in odds [31].

Investigators further assessed racial disparity regarding HIV infection among MSM. In their uncorrected model, MSM were approximately 10 times as likely $(95 \%$ CI: $5.5,18)$ to be infected with HIV compared to non-MSM [30]. Further stratification by race demonstrated that White MSM had greater odds of HIV infection (adjusted prevalence OR $=16$; 95\% CI: 7.4, 34) compared to non-MSM, whereas Black MSM were 4.5 times as likely $(95 \%$ CI: 1.4, 12) to acquire HIV compared to non-MSM (a four-fold difference between these racial groups) [30]. After adjusting for misclassification, differences in HIV risk between White and Black MSM in the uncorrected model reduced from a factor of four to two [30]. These results indicated that additional analytic procedures are necessary when using proxies for the exposure (e.g., sexual identity) and outcome (e.g., self-reported HIV infection). 
Table 1. Selected examples of HIV research among men who have sex with men (MSM) and adjustment methods for purported misclassification of sexual behaviors.

\begin{tabular}{|c|c|c|c|c|c|c|c|c|c|}
\hline \multirow{2}{*}{ Reference } & \multirow{2}{*}{ Data Source } & \multirow{2}{*}{ Study Description } & \multirow{2}{*}{$\begin{array}{c}\text { Population-Based vs. } \\
\text { Community-Based Survey }\end{array}$} & \multirow{2}{*}{$\begin{array}{c}\text { Survey } \\
\text { Administration }\end{array}$} & \multicolumn{2}{|c|}{ Proxy Variables } & \multicolumn{2}{|c|}{ Behavioral Variables } & \multirow[t]{2}{*}{$\begin{array}{c}\text { Treatment of } \\
\text { Misclassification }\end{array}$} \\
\hline & & & & & $\begin{array}{c}\text { Sexual } \\
\text { Identify }\end{array}$ & $\begin{array}{l}\text { Partner } \\
\text { Gender }\end{array}$ & $\begin{array}{c}\text { Condomless } \\
\text { Anal Intercourse }\end{array}$ & Position & \\
\hline Cai et al., 2012 [16] & $\begin{array}{l}\text { Cross-sectional survey } \\
\text { administered in } \\
\text { Shenzhen, China, } 2008\end{array}$ & $\begin{array}{c}\text { Cross-sectional analysis } \\
\text { assessing factors associated } \\
\text { with HIV prevalence among } \\
\text { male sex workers }\end{array}$ & Community-based Survey & $\begin{array}{l}\text { Self-administered } \\
\text { questionnaire }\end{array}$ & & & $\mathrm{x}$ & $\mathrm{x}$ & $\begin{array}{l}\text { Acknowledgement, } \\
\text { but no adjustment }\end{array}$ \\
\hline Ko et al., 2011 [15] & $\begin{array}{l}\text { Community-based } \\
\text { cross-sectional survey, } \\
\text { Taiwan, 2004-2008 }\end{array}$ & $\begin{array}{l}\text { Cross-sectional analysis of } \\
\text { factors associated } \\
\text { with HIV/STI } \\
\end{array}$ & Community-based Survey & $\begin{array}{l}\text { Self-administered } \\
\text { questionnaire }\end{array}$ & & & $\mathrm{x}$ & & $\begin{array}{l}\text { Acknowledgement, } \\
\text { but no adjustment }\end{array}$ \\
\hline Joseph et al., 2011 [32] & $\begin{array}{l}\text { Brothers y Hermanos } \\
\text { Study, 2005-2006 }\end{array}$ & $\begin{array}{c}\text { Cross-sectional analysis of } \\
\text { risk for unrecognized HIV } \\
\text { infection among Black and } \\
\text { Latino MSM } \\
\end{array}$ & Community-based Survey & $\begin{array}{l}\text { Self-administered } \\
\text { computer-based } \\
\text { questionnaire }\end{array}$ & & & $\mathrm{x}$ & $\mathrm{x}$ & $\begin{array}{l}\text { Acknowledgement, } \\
\text { but no adjustment }\end{array}$ \\
\hline Oster et al., 2011 [33] & $\begin{array}{l}\text { National HIV Behavioral } \\
\text { Surveillance Survey } \\
\text { (NHBS), } 2008\end{array}$ & $\begin{array}{l}\text { Cross-sectional CI analysis } \\
\text { assessing disparities in HIV } \\
\text { infection between } \\
\text { racial groups }\end{array}$ & Population-based Survey & $\begin{array}{c}\text { Interviewer } \\
\text { administered } \\
\text { computer-based } \\
\text { questionnaire }\end{array}$ & & & $x$ & & None \\
\hline Reisner et al., 2011 [34] & $\begin{array}{l}\text { National Epidemiologic } \\
\text { Survey on Alcohol and } \\
\text { Related Conditions } \\
\text { (NESARC), 2004-2005 }\end{array}$ & $\begin{array}{l}\text { Cross-sectional analysis } \\
\text { assessing the association } \\
\text { between HIV infection and } \\
\text { early life stressors among } \\
\text { MSM using a national survey }\end{array}$ & Population-based Survey & $\begin{array}{l}\text { Interviewer } \\
\text { administered } \\
\text { computer-based } \\
\text { questionnaire }\end{array}$ & & $x$ & & & $\begin{array}{l}\text { Acknowledgement, } \\
\text { but no adjustment }\end{array}$ \\
\hline Walker et al., 2011 [27] & $\begin{array}{c}\text { Gay men sexual health } \\
\text { survey (GMSHS), British } \\
\text { National Survey of } \\
\text { Sexual Attitudes and } \\
\text { Lifestyles (NATSAL), } \\
\text { genitourinary medicine } \\
\text { (GUM) clinic }\end{array}$ & $\begin{array}{l}\text { Cross-sectional analysis } \\
\text { estimating undiagnosed HIV } \\
\text { infection among MSM }\end{array}$ & $\begin{array}{c}\text { Population- and } \\
\text { Community-based Survey }\end{array}$ & $\begin{array}{l}\text { Self-administered } \\
\text { questionnaire; } \\
\text { self-administered } \\
\text { computer-based } \\
\text { questionnaire; disease } \\
\text { reporting system } \\
\end{array}$ & & & $\mathrm{x}$ & & $\begin{array}{l}\text { Acknowledgement, } \\
\text { but no adjustment }\end{array}$ \\
\hline Zhong et al., 2011 [19] & $\begin{array}{l}\text { Cross-sectional survey, } \\
\text { Guangzhou, China, } 2008\end{array}$ & $\begin{array}{l}\text { Cross-sectional analysis } \\
\text { assessing prevalence of HIV } \\
\text { among MSM }\end{array}$ & Population-based Survey & $\begin{array}{l}\text { Interviewer } \\
\text { administered } \\
\text { questionnaire }\end{array}$ & & & $\mathrm{x}$ & $\mathrm{x}$ & $\begin{array}{l}\text { Acknowledgement, } \\
\text { but no adjustment }\end{array}$ \\
\hline Sweet et al., 2012 [43] & $\begin{array}{l}\text { National Epidemiologic } \\
\text { Survey on Alcohol and } \\
\text { Related Conditions } \\
\text { (NESARC), 2004-2005 }\end{array}$ & $\begin{array}{l}\text { Cross-sectional analysis } \\
\text { assessing the association } \\
\text { between HIV risk and CSA }\end{array}$ & Population-based Survey & $\begin{array}{c}\text { Interviewer } \\
\text { administered } \\
\text { computer-based } \\
\text { questionnaire }\end{array}$ & $\mathrm{x}$ & $\mathrm{x}$ & & & $\begin{array}{l}\text { Acknowledgement, } \\
\text { but no adjustment }\end{array}$ \\
\hline Wang et al., 2012 [18] & $\begin{array}{l}\text { Community based } \\
\text { survey, Harbin, } \\
\text { China, 2006-2010 }\end{array}$ & $\begin{array}{l}\text { Cross-section analysis of HIV } \\
\text { prevalence/syphilis and the } \\
\text { context of lower rates of } \\
\text { condomless anal sex }\end{array}$ & Community-based Survey & $\begin{array}{l}\text { Interviewer } \\
\text { administered } \\
\text { questionnaire }\end{array}$ & & & $\mathrm{x}$ & & $\begin{array}{l}\text { Acknowledgement, } \\
\text { but no adjustment }\end{array}$ \\
\hline
\end{tabular}


Table 1. Cont

\begin{tabular}{|c|c|c|c|c|c|c|c|c|c|}
\hline \multirow{2}{*}{ Reference } & \multirow{2}{*}{ Data Source } & \multirow{2}{*}{ Study Description } & \multirow{2}{*}{$\begin{array}{l}\text { Population-Based vs. } \\
\text { Community-Based Survey }\end{array}$} & \multirow{2}{*}{$\begin{array}{c}\text { Survey } \\
\text { Administration }\end{array}$} & \multicolumn{2}{|c|}{ Proxy Variables } & \multicolumn{2}{|c|}{ Behavioral Variables } & \multirow{2}{*}{$\begin{array}{c}\text { Treatment of } \\
\text { Misclassification }\end{array}$} \\
\hline & & & & & $\begin{array}{c}\text { Sexual } \\
\text { Identify }\end{array}$ & $\begin{array}{l}\text { Partner } \\
\text { Gender }\end{array}$ & $\begin{array}{c}\text { Condomless } \\
\text { Anal Intercourse }\end{array}$ & Position & \\
\hline Balaji et al., 2013 [36] & $\begin{array}{l}\text { National HIV Behavioral } \\
\text { Surveillance Survey } \\
\text { (NHBS), } 2008\end{array}$ & $\begin{array}{l}\text { Cross-sectional analysis } \\
\text { assessing factors associated } \\
\text { with HIV incidence } \\
\text { and prevalence }\end{array}$ & Population-based Survey & $\begin{array}{c}\text { Interviewer } \\
\text { administered } \\
\text { computer-based } \\
\text { questionnaire }\end{array}$ & & & $x$ & $x$ & $\begin{array}{l}\text { Acknowledgement, } \\
\text { but no adjustment }\end{array}$ \\
\hline Konda et al., 2013 [24] & $\begin{array}{c}\text { National Institute of } \\
\text { Mental Health (NIMH) } \\
\text { Collaborative HIV /STD } \\
\text { Prevention Trial Group, } \\
\text { 2002-2007 }\end{array}$ & $\begin{array}{l}\text { Longitudinal analysis of } \\
\text { factors associated } \\
\text { with HIV /STI }\end{array}$ & Community-based survey & $\begin{array}{l}\text { Interviewer } \\
\text { administered } \\
\text { questionnaire }\end{array}$ & & $\mathrm{x}$ & $\mathrm{x}$ & & $\begin{array}{l}\text { Acknowledgement, } \\
\text { but no adjustment }\end{array}$ \\
\hline Sanders et al., 2013 [40] & $\begin{array}{l}\text { Prospective cohort study } \\
\text { assessing sexual } \\
\text { behaviors and } \\
\text { HIV /STI acquisition }\end{array}$ & $\begin{array}{l}\text { Longitudinal analysis } \\
\text { assessing factors for } \\
\text { HIV infection }\end{array}$ & Community-based Survey & $\begin{array}{l}\text { Interviewer } \\
\text { administered } \\
\text { questionnaire }\end{array}$ & & & $\mathrm{x}$ & $x$ & $\begin{array}{l}\text { Acknowledgement, } \\
\text { but no adjustment }\end{array}$ \\
\hline Tafuma et al., 2014 [22] & $\begin{array}{l}\text { Ministry of Health and } \\
\text { Family Health } \\
\text { International } 360 \text { Survey, } \\
\text { Botswana, } 2012 \\
\end{array}$ & $\begin{array}{l}\text { Cross-sectional survey } \\
\text { analysis assessing factors } \\
\text { associated with HIV/STI }\end{array}$ & Population-based Survey & $\begin{array}{l}\text { Interviewer } \\
\text { administered } \\
\text { questionnaire }\end{array}$ & & & $x$ & $x$ & $\begin{array}{l}\text { Acknowledgement, } \\
\text { but no adjustment }\end{array}$ \\
\hline van den Boom et al., 2014 [26] & $\begin{array}{l}\text { Amsterdam Cohort } \\
\text { Study (ACS), 2007-2011 }\end{array}$ & $\begin{array}{l}\text { Cross-sectional survey } \\
\text { analysis assessing factors } \\
\text { associated with HIV }\end{array}$ & Population-based Survey & $\begin{array}{l}\text { Self-administered } \\
\text { questionnaire }\end{array}$ & & & $x$ & $\mathrm{x}$ & $\begin{array}{l}\text { Acknowledgement, } \\
\text { but no adjustment }\end{array}$ \\
\hline Castillo et al., 2015 [25] & $\begin{array}{c}\text { Longitudinal study } \\
\text { assessing sexual } \\
\text { behaviors and HIV/STI } \\
\text { acquisition at baseline, 9- } \\
\text { and 18-month follow-up }\end{array}$ & $\begin{array}{l}\text { Discrete time proportional } \\
\text { hazard models assessing } \\
\text { factors associated with } \\
\text { incidence of HIV/STI }\end{array}$ & Community-based Survey & $\begin{array}{l}\text { Interviewer } \\
\text { administered } \\
\text { questionnaire }\end{array}$ & & & $x$ & $x$ & $\begin{array}{l}\text { Acknowledgement, } \\
\text { but no adjustment }\end{array}$ \\
\hline Crosby et al., 2015 [41] & $\begin{array}{c}\text { Convenience sample } \\
\text { recruited from National } \\
\text { Institute of Health-RCT } \\
\text { for safer sex intervention, } \\
\text { 2012-2014 }\end{array}$ & $\begin{array}{l}\text { Cross-sectional analysis } \\
\text { assessing differences } \\
\text { between HIV-positive and } \\
\text { negative MSM in regard to } \\
\text { condom use }\end{array}$ & Community-based Survey & $\begin{array}{l}\text { Self-administered } \\
\text { questionnaire }\end{array}$ & & & $x$ & $x$ & $\begin{array}{l}\text { Acknowledgement, } \\
\text { but no adjustment }\end{array}$ \\
\hline Dailey Garnes et al., 2015 [46] & $\begin{array}{l}\text { Sexually Transmitted } \\
\text { Disease Management } \\
\text { Information System } \\
\text { (STD-MIS) }\end{array}$ & $\begin{array}{l}\text { Cross-sectional analysis } \\
\text { assessing odds of identifying } \\
\text { new HIV infection among } \\
\text { social contacts using } \\
\text { surveillance data }\end{array}$ & Neither & $\begin{array}{c}\text { Disease reporting } \\
\text { system }\end{array}$ & & $x$ & & & $\begin{array}{l}\text { Acknowledgement, } \\
\text { but no adjustment }\end{array}$ \\
\hline Halkitis et al., 2015 [38] & $\begin{array}{l}\text { Prospective cohort study } \\
\text { assessing factors } \\
\text { associated with } \\
\text { HIV infection }\end{array}$ & $\begin{array}{l}\text { Survival analysis assessing } \\
\text { factors associated with HIV } \\
\text { incidence among young } \\
\text { MSM (18-19 years) }\end{array}$ & Community-based Survey & $\begin{array}{l}\text { Interviewer } \\
\text { administered } \\
\text { questionnaire }\end{array}$ & & & $\mathrm{x}$ & $\mathrm{x}$ & None \\
\hline
\end{tabular}


Table 1. Cont

\begin{tabular}{|c|c|c|c|c|c|c|c|c|c|}
\hline \multirow{2}{*}{ Reference } & \multirow{2}{*}{ Data Source } & \multirow{2}{*}{ Study Description } & \multirow{2}{*}{$\begin{array}{l}\text { Population-Based vs. } \\
\text { Community-Based Survey }\end{array}$} & \multirow{2}{*}{$\begin{array}{c}\text { Survey } \\
\text { Administration }\end{array}$} & \multicolumn{2}{|c|}{ Proxy Variables } & \multicolumn{2}{|c|}{ Behavioral Variables } & \multirow{2}{*}{$\begin{array}{c}\text { Treatment of } \\
\text { Misclassification }\end{array}$} \\
\hline & & & & & $\begin{array}{c}\text { Sexual } \\
\text { Identify }\end{array}$ & $\begin{array}{l}\text { Partner } \\
\text { Gender }\end{array}$ & $\begin{array}{c}\text { Condomless } \\
\text { Anal Intercourse }\end{array}$ & Position & \\
\hline Qian et al., 2015 [20] & $\begin{array}{l}\text { Cross-sectional survey, } \\
\text { Beijing, China, 2010-2011 }\end{array}$ & $\begin{array}{l}\text { Cross-sectional analysis } \\
\text { assessing risk factors } \\
\text { associated with } \\
\text { HIV prevalence } \\
\end{array}$ & Community-based Survey & $\begin{array}{l}\text { Self-administered } \\
\text { questionnaire }\end{array}$ & & & & $\mathrm{x}$ & $\begin{array}{l}\text { Acknowledgement, } \\
\text { but no adjustment }\end{array}$ \\
\hline Solomon et al., 2015 [17] & $\begin{array}{l}\text { Multicenter } \\
\text { cross-sectional } \\
\text { survey, } 12 \text { cities }\end{array}$ & $\begin{array}{l}\text { Cross-sectional analysis } \\
\text { assessing prevalence, } \\
\text { incidence and factors } \\
\text { associated with } \\
\text { HIV infection }\end{array}$ & Population-based Survey & $\begin{array}{l}\text { Interviewer } \\
\text { administered } \\
\text { computer-based } \\
\text { questionnaire }\end{array}$ & & & $\mathrm{x}$ & $\mathrm{x}$ & $\begin{array}{l}\text { Acknowledgement, } \\
\text { but no adjustment }\end{array}$ \\
\hline Sullivan et al., 2015 [35] & $\begin{array}{l}\text { Prospective cohort study, } \\
\text { Atlanta, GA, 2010-2014 }\end{array}$ & $\begin{array}{l}\text { Survival analysis assessing } \\
\text { racial disparities in } \\
\text { HIV infection }\end{array}$ & Community-based Survey & $\begin{array}{l}\text { Self-administered } \\
\text { computer-based } \\
\text { questionnaire }\end{array}$ & & & $x$ & $\mathrm{x}$ & $\begin{array}{l}\text { Acknowledgement, } \\
\text { but no adjustment }\end{array}$ \\
\hline Beymer et al., 2016 [28] & $\begin{array}{l}\text { Longitudinal study } \\
\text { assessing HIV risk } \\
\text { factors using a } \\
\text { community-based } \\
\text { survey, Los Angeles, } \\
\text { CA, 2009-2014 }\end{array}$ & $\begin{array}{l}\text { Survival analysis assessing } \\
\text { factors associated with } \\
\text { HIV infection }\end{array}$ & Community-based Survey & $\begin{array}{l}\text { Interviewer } \\
\text { administered } \\
\text { questionnaire }\end{array}$ & & & $x$ & $\mathrm{x}$ & None \\
\hline Eaton et al., 2016 [29] & $\begin{array}{l}\text { Cross-sectional survey of } \\
\text { Black MSM }\end{array}$ & $\begin{array}{l}\text { Cross-sectional analysis } \\
\text { assessing factors associated } \\
\text { with testing HIV positive }\end{array}$ & Community-based Survey & $\begin{array}{l}\text { Self-administered } \\
\text { computer-based } \\
\text { questionnaire }\end{array}$ & & & $\mathrm{x}$ & & $\begin{array}{l}\text { Acknowledgement, } \\
\text { but no adjustment }\end{array}$ \\
\hline Garofalo et al., 2016 [37] & $\begin{array}{l}\text { Crew 45-Longitudinal } \\
\text { study assessing HIV risk } \\
\text { in Chicago for } 2 \text { years }\end{array}$ & $\begin{array}{l}\text { Survival analysis assessing } \\
\text { factors associated with HIV } \\
\text { incidence among young } \\
\text { MSM (16-20 years) }\end{array}$ & Community-based Survey & $\begin{array}{l}\text { Self-administered } \\
\text { computer-based } \\
\text { questionnaire }\end{array}$ & & & $\mathrm{x}$ & & $\begin{array}{l}\text { Acknowledgement, } \\
\text { but no adjustment }\end{array}$ \\
\hline Khosropour et al., 2016 [44] & $\begin{array}{l}\text { Data from STD clinic in } \\
\text { Seattle \& King County, } \\
\text { 2001-2013 }\end{array}$ & $\begin{array}{l}\text { Retrospective case-control } \\
\text { study assessing factors } \\
\text { associated with } \\
\text { seroconversion and } \\
\text { difference in sexual behavior } \\
\text { after seroconversion }\end{array}$ & Population-based Survey & $\begin{array}{l}\text { Disease reporting } \\
\text { system }\end{array}$ & & & $\mathrm{x}$ & & $\begin{array}{l}\text { Acknowledgement, } \\
\text { but no adjustment }\end{array}$ \\
\hline Davey et al., 2017 [42] & $\begin{array}{l}\text { Retrospective study of } \\
\text { HIV testing data from LA } \\
\text { LGBT Center, 2011-2015 }\end{array}$ & $\begin{array}{l}\text { Cross-sectional analysis of } \\
\text { MSM assessing factors } \\
\text { associated with acute HIV } \\
\text { infection } \\
\end{array}$ & Community-based Survey & $\begin{array}{l}\text { Interviewer } \\
\text { administered } \\
\text { questionnaire }\end{array}$ & & & $\mathrm{x}$ & $\mathrm{x}$ & $\begin{array}{l}\text { Acknowledgement, } \\
\text { but no adjustment }\end{array}$ \\
\hline German et al., 2017 [45] & $\begin{array}{l}\text { National HIV Behavioral } \\
\text { Surveillance Survey } \\
\text { (NHBS), } 2008\end{array}$ & $\begin{array}{l}\text { Cross-sectional analysis of } \\
\text { factors associated with HIV } \\
\text { transmission risk }\end{array}$ & Population-based Survey & $\begin{array}{l}\text { Interviewer } \\
\text { administered } \\
\text { computer-based } \\
\text { questionnaire }\end{array}$ & & & $x$ & $x$ & $\begin{array}{l}\text { Acknowledgement, } \\
\text { but no adjustment }\end{array}$ \\
\hline
\end{tabular}


Table 1. Cont

\begin{tabular}{|c|c|c|c|c|c|c|c|c|c|}
\hline \multirow{2}{*}{ Reference } & \multirow{2}{*}{ Data Source } & \multirow{2}{*}{ Study Description } & \multirow{2}{*}{$\begin{array}{l}\text { Population-Based vs. } \\
\text { Community-Based Survey }\end{array}$} & \multirow{2}{*}{$\begin{array}{c}\text { Survey } \\
\text { Administration }\end{array}$} & \multicolumn{2}{|c|}{ Proxy Variables } & \multicolumn{2}{|c|}{ Behavioral Variables } & \multirow{2}{*}{$\begin{array}{c}\text { Treatment of } \\
\text { Misclassification }\end{array}$} \\
\hline & & & & & $\begin{array}{l}\text { Sexual } \\
\text { Identify }\end{array}$ & $\begin{array}{l}\text { Partner } \\
\text { Gender }\end{array}$ & $\begin{array}{c}\text { Condomless } \\
\text { Anal Intercourse } \\
\end{array}$ & Position & \\
\hline Goldstein et al., 2015 [31] & $\begin{array}{l}\text { National Epidemiologic } \\
\text { Survey on Alcohol and } \\
\text { Related Conditions } \\
\text { (NESARC-2) \& Black and } \\
\text { African American Men's } \\
\text { Health Study (BAAMHS) }\end{array}$ & $\begin{array}{l}\text { Bayesian analysis for the } \\
\text { odds of self-reported HIV } \\
\text { infection when adjusted for } \\
\text { misclassification of } \\
\text { same-sex behavior }\end{array}$ & $\begin{array}{c}\text { Population- and } \\
\text { Community-based Survey }\end{array}$ & $\begin{array}{c}\text { Interviewer } \\
\text { administered } \\
\text { computer-based } \\
\text { questionnaire; } \\
\text { self-administered } \\
\text { computer-based } \\
\text { questionnaire }\end{array}$ & $x$ & $\mathrm{x}$ & $\mathrm{x}$ & $\mathrm{x}$ & $\begin{array}{l}\text { Acknowledgement } \\
\text { and Bayesian } \\
\text { adjustment }\end{array}$ \\
\hline Goldstein et al., 2017 [30] & $\begin{array}{l}\text { National Epidemiologic } \\
\text { Survey on Alcohol and } \\
\text { Related Conditions } \\
\text { (NESARC-2) \& Black and } \\
\text { African American Men's } \\
\text { Health Study (BAAMHS) }\end{array}$ & $\begin{array}{l}\text { Bayesian analysis to adjust } \\
\text { for residual confounding and } \\
\text { correct misclassification of } \\
\text { MSM status to help explain } \\
\text { racial disparity in } \\
\text { HIV infection }\end{array}$ & $\begin{array}{c}\text { Population- and } \\
\text { Community-based Survey }\end{array}$ & $\begin{array}{c}\text { Interviewer } \\
\text { administered } \\
\text { computer-based } \\
\text { questionnaire; } \\
\text { self-administered } \\
\text { computer-based } \\
\text { questionnaire }\end{array}$ & $x$ & $\mathrm{x}$ & $x$ & $\mathrm{x}$ & $\begin{array}{l}\text { Acknowledgement } \\
\text { and Bayesian } \\
\text { adjustment }\end{array}$ \\
\hline Kunzweiler et al., 2017 [21] & $\begin{array}{l}\text { The Anza Mapema } \\
\text { Study, 2015-2016 }\end{array}$ & $\begin{array}{l}\text { Cross-sectional analysis of } \\
\text { risk reduction behaviors } \\
\text { associated with HIV } \\
\text { prevalence among } \\
\text { HIV-positive and out of care } \\
\text { (vs. HIV negative) and newly } \\
\text { diagnosed HIV positive } \\
\text { and out of care }\end{array}$ & Community-based Survey & $\begin{array}{l}\text { Self-administered } \\
\text { computer-based } \\
\text { questionnaire }\end{array}$ & & & $\mathrm{x}$ & & $\begin{array}{l}\text { Acknowledgement, } \\
\text { but no adjustment }\end{array}$ \\
\hline Marano et al., 2017 [39] & $\begin{array}{l}\text { NHM\&E-National HIV } \\
\text { Prevention Program } \\
\text { Monitoring and } \\
\text { Evaluation system, } 2015\end{array}$ & $\begin{array}{l}\text { Cross-sectional analysis } \\
\text { describing linkage to care } \\
\text { among new HIV diagnoses } \\
\text { and assessing factors } \\
\text { associated with } \\
\text { HIV incidence }\end{array}$ & Population-based Survey & $\begin{array}{l}\text { Disease reporting } \\
\text { system }\end{array}$ & & & $\mathrm{x}$ & & None \\
\hline Wirtz et al., 2017 [23] & $\begin{array}{c}\text { Multi-center } \\
\text { cross-sectional } \\
\text { survey, 2010-2014 }\end{array}$ & $\begin{array}{l}\text { Cross-sectional analysis } \\
\text { assessing regional disparities } \\
\text { in HIV prevalence and care }\end{array}$ & Population-based Survey & $\begin{array}{l}\text { Interviewer } \\
\text { administered } \\
\text { questionnaire }\end{array}$ & & & $\mathrm{x}$ & & $\begin{array}{l}\text { Acknowledgement, } \\
\text { but no adjustment }\end{array}$ \\
\hline
\end{tabular}




\section{Discussion}

\subsection{Correcting Risk Estimates}

Recent work has summarized techniques for correcting misclassification bias for exposure estimates related to HIV epidemiology [47-49]. Goldstein et al. [30,31] demonstrated that using proxy variables may result in inaccurate risk estimation of HIV infection and how risk-adjusting methodologies are an attractive option to arrive at improved assessment of risk when dealing with potentially misclassified risk data that have been previously collected. Furthermore, these techniques demonstrate an intersection of epidemiological methodology and public health practice: research is not occurring in a vacuum but has real-world implications.

We observed that the majority of reviewed studies did not rely upon proxy exposures, but rather asked specific behavioral questions. Thus, the magnitude of the misclassification may not be as dramatic as first hypothesized. Nevertheless, the potential for misclassification remains as these are observational data. Considering that asking someone intimate details about their sexual acts can lead to prevarication to obscure potentially behaviors, underreporting may lead to biased HIV risk estimation [7,8]. Further, the manner in which questions are asked may elicit varying degrees of misclassification. For example, having an interviewer administer the questions may invoke a social desirability bias, unless trust has been established between the interviewee and interviewer. Therefore, even if the appropriate behavioral questions are asked (with respect to risk for HIV acquisition), there is potential for information bias, which warrants careful consideration by the investigators and use of bias adjustment techniques.

\subsection{Implications of Biased HIV Risk Data}

Consider that an urban health department had data on the HIV epidemic solely from a national survey that reported sexual identity as a proxy for high-risk sexual behavior. The analysis from this survey may demonstrate a strong association between identifying as gay or bisexual and reported HIV infection. Thus, the health department targets its limited funding and resources for HIV testing and prevention campaigns into the city's gay neighborhood, where the majority of self-identifying lesbian, gay, bisexual, and transgender people live or socialize [50]. While this will undoubtedly have impact, it will also miss populations with substantial risk for infection. The corrected estimates speculate at what might be had the survey included behavioral data, but the analytic methods outlined above cannot elucidate new characteristics.

Now consider that a health department has data from a survey that has included assessment of specific sexual behaviors in addition to nominal risk group information indicated by self-reported sexual orientation. The analysis may suggest that in addition to self-identified gay men as having an increased risk, there are also pockets of racial minority MSM that have a substantial risk of infection. However, as MSM of color face greater stigma and are less likely to disclose same-sex behavior [51], this underreporting may mask the HIV risk disparity. The use of quantitative bias analysis can assess the degree to which this misclassification is affecting the risk estimates [10]. However, bias correction is not always critical to good policy just as the result of one epidemiological study may never be the sole basis for policy decisions. Observational studies are one of many potential considerations for policy implementation and may sometimes be beneficial to engage in bias adjustment as it provides assurance for the investigators of the internal validity of their analyses.

\subsection{Towards Improving the Data}

We also use this systematic review as a call to those engaging in primary data collection within sexual minority populations to include detailed behavioral assessment. For example, consider survey items from NESARC, one potential source of nationally generalizable data for HIV infection among sexual minorities [43]. MSM behavior can be inferred from two survey items: one collecting information about self-reported sexual orientation, and a second asking about whether survey respondents have 
had sex with men. Specific sexual acts are not queried; if a participant indicated he had sex with other men, the risk for HIV is highly dependent upon sexual acts (anal as opposed to oral) and positioning (receptive for greatest risk). A prototypical example comes from the Black and African American Men's Health Study (BAAMHS): in addition to data on reported sexual identity and partner gender, specific sexual behaviors were also obtained, and while these data more likely ascribe an accurate risk profile, they are limited to non-monogamous black men in the Boston area [52].

To improve internal and external validity of findings for HIV and STI evaluations, newer studies are needed with the same level of detail on sexual behaviors as BAAMHS, including: (1) frequency of specific sexual behaviors (oral and/or anal intercourse); (2) positioning (receptive and/or insertive); (3) HIV testing and treatment status; (4) engaging in seroadaptive sex practices, including discussion of HIV infection status and changes of behaviors based on this discussion, including condom use; and (5) information about partnerships to assess sexual networks. Assessing stigmatizing behaviors and conditions via surveys further requires careful consideration for how data are collected in the field. In NESARC, the survey was administered in participants' homes with research personnel and perhaps even household members present. This is less than ideal, since ensuring some level of privacy for respondents can mitigate underreporting and the social desirability bias leading to differential misclassification of sexual orientation and behaviors.

Our assessment of recent studies indicates questions pertaining to sexual behavior are more common than proxy exposures, encouraging for HIV risk estimation among sexual minority men. However, as discussed there is still the opportunity for risk estimation to be improved by considering underreporting of risk data. Missing or messy data may potentially fail to provide adequate understanding of the relationship between sexuality and HIV risk. Thus, ascertaining more nuanced information on sexual behaviors and conducting appropriate analyses are needed to make more informed estimation. Yet we also acknowledge the importance of proxy variables such as sexual orientation. These may be appropriate for assessing certain socio-behavioral constructs such as perceived discrimination, stigma, and HIV screening attitude among self-identified gay/bisexual men.

Our systematic review is subjected to some limitations. It is possible that our search methodology may have missed articles that are pertinent to our aims in describing methods of capturing high risk sexual behaviors. However, the journals that we have selected are high impact journals, thus our results highlight current efforts in the field of HIV risk research and bias correction methods to accurately measure high risk sexual behavior. Secondly, we did not assess misclassification of HIV infection in our review. We understand that measurement error and social desirability bias have the same potential of impacting HIV infection. Previous studies have explored potential solutions for such biases [27,48]. Finally, we are limited to reporting only adjustment techniques that have been presented in the articles. It is possible that investigators are engaging in these analytic methods. If so, we may be underreporting the use of bias analysis techniques. However, absence of misclassification correction techniques should also not be viewed as biased HIV risk estimates.

\section{Conclusions}

While the call for better exposure assessment may not be new [6,11], recent evidence of the magnitude of its bias on HIV transmission risk indicates this is a public health concern that remains. Given the predetermined limitations of how survey items may be collected, analytic methodologies can provide an approach to correct misclassification bias around sexuality and sexual behaviors. However, these techniques can only do so much in the presence of imperfect data. Rather than devoting substantial time and resources to methods to improving data analysis, we should improve the quality of data collected, through more accurate assessment of specific sexual behaviors. In short, it is high-risk behaviors (i.e., receptive anal intercourse) which confer the greatest risk; therefore, questions surrounding sexual behavior (i.e., sexual act and positioning) are of primary importance in surveys that include sexual minority populations. 
Author Contributions: S.L.W and N.D.G developed the concept for this paper and the approach used for this analysis; N.K.T. performed the systematic review; N.K.T. and N.D.G. wrote the manuscript; S.L.W. contributed to reviewing and editing the manuscript.

Funding: No funding was obtained for this work.

Acknowledgments: The authors would like to thank Lezhou $\mathrm{Wu}$ and Dina Teryoleva for their assistance in conducting the systematic review.

Conflicts of Interest: The authors report no conflicts.

\section{References}

1. Durso, L.E.; Gates, G.J. Best practices: Collecting and analyzing data on sexual minorities. In International Handbook on the Demography of Sexuality; Baumle, A.K., Ed.; Springer: Dordrecht, The Netherlands, 2013; pp. 21-42.

2. Richters, J. Understanding sexual orientation: A plea for clarity. Reprod. Health Matters 1998, 6, $144-149$. [CrossRef]

3. Beyrer, C.; Baral, S.D.; van Griensven, F.; Goodreau, S.M.; Chariyalertsak, S.; Wirtz, A.L.; Brookmeyer, R. Global epidemiology of hiv infection in men who have sex with men. Lancet 2012, 380, 367-377. [CrossRef]

4. Institute of Medicine of the National Academies. The Health of Lesbian, Gay, Bisexual, and Transgender People: Building a Foundation for Better Understanding; The National Academies Press: Washington, DC, USA, 2011.

5. McFarland, W.; Caceres, C.F. HIV surveillance among men who have sex with men. AIDS 2001, 15, S23-S32. [CrossRef] [PubMed]

6. Pathela, P.; Hajat, A.; Schillinger, J.; Blank, S.; Sell, R.; Mostashari, F. Discordance between sexual behavior and self-reported sexual identity: A population-based survey of new york city men. Ann. Intern. Med. 2006, 145, 416-425. [CrossRef] [PubMed]

7. Latkin, C.; Yang, C.; Tobin, K.; Roebuck, G.; Spikes, P.; Patterson, J. Social network predictors of disclosure of msm behavior and hiv-positive serostatus among african american msm in baltimore, maryland. AIDS Behav. 2012, 16, 535-542. [CrossRef] [PubMed]

8. Zea, M.C.; Reisen, C.A.; Poppen, P.J.; Echeverry, J.J.; Bianchi, F.T. Disclosure of hiv-positive status to latino gay men's social networks. Am. J. Community Psychol. 2004, 33, 107-116. [CrossRef] [PubMed]

9. Savin-Williams, R.C. Who's gay? Does it matter? Curr. Dir. Psychol. Sci. 2006, 15, 40-44. [CrossRef]

10. Lash, T.L.; Fink, A.K.; Fox, M.P. Misclassification. In Applying Quantitative Bias Analysis to Epidemiologic Data; Springer: New York, NY, USA, 2009; pp. 79-108.

11. Igartua, K.; Thombs, B.D.; Burgos, G.; Montoro, R. Concordance and discrepancy in sexual identity, attraction, and behavior among adolescents. J. Adolesc. Health 2009, 45, 602-608. [CrossRef] [PubMed]

12. Moher, D.; Liberati, A.; Tetzlaff, J.; Altman, D.G. Preferred reporting items for systematic reviews and meta-analyses: The prisma statement. PLoS Med. 2009, 6, e1000097. [CrossRef] [PubMed]

13. Klebanoff, M.A.; Cole, S.R. Use of multiple imputation in the epidemiologic literature. Am. J. Epidemiol. 2008, 168, 355-357. [CrossRef] [PubMed]

14. Brakenhoff, T.B.; Mitroiu, M.; Keogh, R.H.; Moons, K.G.M.; Groenwold, R.H.H.; van Smeden, M. Measurement error is often neglected in medical literature: A systematic review. J. Clin. Epidemiol. 2018, 98, 89-97. [CrossRef] [PubMed]

15. Ko, N.Y.; Lee, H.C.; Hung, C.C.; Tseng, F.C.; Chang, J.L.; Lee, N.Y.; Chang, C.M.; Lee, M.P.; Chen, B.J.; Wang, S.W.; et al. Trends of HIV and sexually transmitted infections, estimated hiv incidence, and risky sexual behaviors among gay bathhouse attendees in taiwan: 2004-2008. AIDS Behav. 2011, 15, $292-297$. [CrossRef] [PubMed]

16. Cai, W.-D.; Zhao, J.; Gan, Y.-X.; Zhang, Y.; Yang, Z.-R.; Cheng, J.-Q.; Lin, S.-H.; He, M.-L.; Chen, L.; Wang, X.-R. A comparison of HIV infection and related risk factors between money boys and noncommercial men who have sex with men in shenzhen, china. Sex. Transm. Dis. 2012, 39, 942-948.

17. Solomon, S.S.; Mehta, S.H.; Srikrishnan, A.K.; Vasudevan, C.K.; McFall, A.M.; Balakrishnan, P.; Anand, S.; Nandagopal, P.; Ogburn, E.L.; Laeyendecker, O.; et al. High HIV prevalence and incidence among msm across 12 cities in india. AIDS 2015, 29, 723-731. [CrossRef] [PubMed] 
18. Wang, K.; Yan, H.; Liu, Y.; Leng, Z.; Wang, B.; Zhao, J. Increasing prevalence of hiv and syphilis but decreasing rate of self-reported unprotected anal intercourse among men who had sex with men in harbin, China: Results of five consecutive surveys from 2006 to 2010. Int. J. Epidemiol. 2012, 41, 423-432. [CrossRef] [PubMed]

19. Zhong, F.; Lin, P.; Xu, H.; Wang, Y.; Wang, M.; He, Q.; Fan, L.; Li, Y.; Wen, F.; Liang, Y.; et al. Possible increase in hiv and syphilis prevalence among men who have sex with men in guangzhou, China: Results from a respondent-driven sampling survey. AIDS Behav. 2011, 15, 1058-1066. [CrossRef] [PubMed]

20. Qian, H.Z.; Ruan, Y.; Liu, Y.; Milam, D.F.; Spiegel, H.M.; Yin, L.; Li, D.; Shepherd, B.E.; Shao, Y.; Vermund, S.H. Lower HIV risk among circumcised men who have sex with men in China: Interaction with anal sex role in a cross-sectional study. J. Acquir. Immune Defic. Syndr. 2016, 71, 444-451. [CrossRef] [PubMed]

21. Kunzweiler, C.P.; Bailey, R.C.; Okall, D.O.; Graham, S.M.; Mehta, S.D.; Otieno, F.O. Factors associated with prevalent HIV infection among Kenyan MSM: The Anza Mapema Study. J. Acquir. Immune Defic. Syndr. 2017, 76, 241-249. [CrossRef] [PubMed]

22. Tafuma, T.A.; Merrigan, M.B.; Okui, L.A.; Lebelonyane, R.; Bolebantswe, J.; Mine, M.; Chishala, S.; Moyo, S.; Thela, T.; Rajatashuvra, A. HIV/sexually transmitted infection prevalence and sexual behavior of men who have sex with men in 3 districts of Botswana: Results from the 2012 biobehavioral survey. Sex. Transm. Infect. 2014, 41, 480-485. [CrossRef] [PubMed]

23. Wirtz, A.L.; Trapence, G.; Kamba, D.; Gama, V.; Chalera, R.; Jumbe, V.; Kumwenda, R.; Mangochi, M.; Helleringer, S.; Beyrer, C.; et al. Geographical disparities in hiv prevalence and care among men who have sex with men in Malawi: Results from a multisite cross-sectional survey. Lancet HIV 2017, 4, e260-e269. [CrossRef]

24. Konda, K.A.; Lescano, A.G.; Celentano, D.D.; Hall, E.; Montano, S.M.; Kochel, T.J.; Coates, T.J.; Cáceres, C.F.; NIMH Collaborative HIVSTD Prevention Trial Group. In Peru, reporting male sex partners imparts significant risk of incident $\mathrm{HIV}$ / sexually transmitted infection: All men engaging in same-sex behavior need prevention services. Sex. Transm. Dis. 2013, 40, 569-574. [CrossRef] [PubMed]

25. Castillo, R.; Konda, K.A.; Leon, S.R.; Silva-Santisteban, A.; Salazar, X.; Klausner, J.D.; Coates, T.J.; Caceres, C.F. $\mathrm{HIV}$ and sexually transmitted infection incidence and associated risk factors among high-risk msm and male-to-female transgender women in Lima, Peru. J. Acquir. Immune Defic. Syndr. 2015, 69, 567-575. [CrossRef] [PubMed]

26. Van den Boom, W.; Konings, R.; Davidovich, U.; Sandfort, T.; Prins, M.; Stolte, I.G. Is serosorting effective in reducing the risk of HIV infection among men who have sex with men with casual sex partners? J. Acquir. Immune Defic. Syndr. 2014, 65, 375-379. [CrossRef] [PubMed]

27. Walker, K.; Seaman, S.R.; De Angelis, D.; Presanis, A.M.; Dodds, J.P.; Johnson, A.M.; Mercey, D.; Gill, O.N.; Copas, A.J. A synthesis of convenience survey and other data to estimate undiagnosed HIV infection among men who have sex with men in England and Wales. Int. J. Epidemiol. 2011, 40, 1358-1366. [CrossRef] [PubMed]

28. Beymer, M.R.; Weiss, R.E.; Halkitis, P.N.; Kapadia, F.; Ompad, D.C.; Bourque, L.; Bolan, R.K. Disparities within the disparity-determining HIV risk factors among latino gay and bisexual men attending a community-based clinic in Los Angeles, CA. J. Acquir. Immune Defic. Syndr. 2016, 73, 237-244. [CrossRef] [PubMed]

29. Eaton, L.A.; Maksut, J.L.; Gamarel, K.E.; Siembida, E.J.; Driffin, D.D.; Baldwin, R. Online sex partner meeting venues as a risk factor for testing HIV positive among a community-based sample of black men who have sex with men. Sex. Transm. Dis. 2016, 43, 360-364. [CrossRef] [PubMed]

30. Goldstein, N.D.; Burstyn, I.; Welles, S.L. Bayesian approaches to racial disparities in HIV risk estimation among men who have sex with men. Epidemiology 2017, 28, 215-220. [CrossRef] [PubMed]

31. Goldstein, N.D.; Welles, S.L.; Burstyn, I. To be or not to be: Bayesian correction for misclassification of self-reported sexual behaviors among men who have sex with men. Epidemiology 2015, 26, 637-644. [CrossRef] [PubMed]

32. Joseph, H.A.; Marks, G.; Belcher, L.; Millett, G.A.; Stueve, A.; Bingham, T.A.; Lauby, J. Older partner selection, sexual risk behaviour and unrecognised HIV infection among black and latino men who have sex with men. Sex. Transm. Infect. 2011, 87, 442-447. [CrossRef] [PubMed] 
33. Oster, A.M.; Wiegand, R.E.; Sionean, C.; Miles, I.J.; Thomas, P.E.; Melendez-Morales, L.; Le, B.C.; Millett, G.A. Understanding disparities in HIV infection between black and white msm in the United States. AIDS 2011, 25, 1103-1112. [CrossRef] [PubMed]

34. Reisner, S.L.; Falb, K.L.; Mimiaga, M.J. Early life traumatic stressors and the mediating role of PTSD in incident HIV infection among US men, comparisons by sexual orientation and race/ethnicity: Results from the Nesarc, 2004-2005. J. Acquir. Immune Defic. Syndr. 2011, 57, 340-350. [CrossRef] [PubMed]

35. Sullivan, P.S.; Rosenberg, E.S.; Sanchez, T.H.; Kelley, C.F.; Luisi, N.; Cooper, H.L.; Diclemente, R.J.; Wingood, G.M.; Frew, P.M.; Salazar, L.F.; et al. Explaining racial disparities in HIV incidence in black and white men who have sex with men in Atlanta, GA: A prospective observational cohort study. Ann. Epidemiol. 2015, 25, 445-454. [CrossRef] [PubMed]

36. Balaji, A.B.; Bowles, K.E.; Le, B.C.; Paz-Bailey, G.; Oster, A.M. High HIV incidence and prevalence and associated factors among young MSM, 2008. AIDS 2013, 27, 269-278. [CrossRef] [PubMed]

37. Garofalo, R.; Hotton, A.L.; Kuhns, L.M.; Gratzer, B.; Mustanski, B. Incidence of HIV infection and sexually transmitted infections and related risk factors among very young men who have sex with men. J. Acquir. Immune Defic. Syndr. 2016, 72, 79-86. [CrossRef] [PubMed]

38. Halkitis, P.; Kapadia, F.; Ompad, D. Incidence of HIV infection in young gay, bisexual, and other YMSM: The p18 cohort study. J. Acquir. Immune Defic. Syndr. 2015, 69, 466-473. [CrossRef] [PubMed]

39. Marano, M.R.; Stein, R.; Williams, W.O.; Wang, G.; Xu, S.; Uhl, G.; Cheng, Q.; Rasberry, C.N. HIV testing in nonhealthcare facilities among adolescent MSM. AIDS 2017, 31 (Suppl. 3), S261-S265. [CrossRef]

40. Sanders, E.J.; Okuku, H.S.; Smith, A.D.; Mwangome, M.; Wahome, E.; Fegan, G.; Peshu, N.; van der Elst, E.M.; Price, M.A.; McClelland, R.S.; et al. High HIV-1 incidence, correlates of HIV-1 acquisition, and high viral loads following seroconversion among MSM. AIDS 2013, 27, 437-446. [CrossRef] [PubMed]

41. Crosby, R.; Mena, L.; Yarber, W.L.; Graham, C.A.; Sanders, S.A.; Milhausen, R.R. Condom use errors and problems: A comparative study of HIV-positive versus HIV-negative young black men who have sex with men. Sex. Transm. Dis. 2015, 42, 634-636. [CrossRef] [PubMed]

42. Davey, D.J.; Beymer, M.; Roberts, C.P.; Bolan, R.K.; Klausner, J.D. Differences in risk behavior and demographic factors between men who have sex with men with acute and nonacute human immunodeficiency virus infection in a community-based testing program in Los Angeles. J. Acquir. Immune Defic. Syndr. 2017, 74, e97-e103. [CrossRef] [PubMed]

43. Sweet, T.; Welles, S.L. Associations of sexual identity or same-sex behaviors with history of childhood sexual abuse and HIV/STI risk in the United States. J. Acquir. Immune Defic. Syndr. 2012, 59, 400-408. [CrossRef] [PubMed]

44. Khosropour, C.M.; Dombrowski, J.C.; Kerani, R.P.; Katz, D.A.; Barbee, L.A.; Golden, M.R. Changes in condomless sex and serosorting among men who have sex with men after HIV diagnosis. J. Acquir. Immune Defic. Syndr. 2016, 73, 475-481. [CrossRef] [PubMed]

45. German, D.; Brady, K.; Kuo, I.; Opoku, J.; Flynn, C.; Patrick, R.; Park, J.N.; Adams, J.; Carroll, M.; Simmons, R.; et al. Characteristics of black men who have sex with men in Baltimore, Philadelphia, and Washington, D.C.: Geographic diversity in socio-demographics and HIV transmission risk. J. Acquir. Immune Defic. Syndr. 2017, 75, S296-S308. [CrossRef] [PubMed]

46. Dailey Garnes, N.J.M.; Moore, Z.S.; Cadwell, B.L.; Fleischauer, A.T.; Leone, P. Previously undiagnosed HIV infections identified through cluster investigation, North Carolina, 2002-2007. AIDS Behav. 2015, 19, 723-731. [CrossRef] [PubMed]

47. Sullivan, P.S.; Rosenberg, E.S. Commentary: Breaking bias: Improved methods for analyzing HIV / AIDS data. Epidemiology 2015, 26, 625-627. [CrossRef] [PubMed]

48. Lash, T.L.; Fox, M.P.; MacLehose, R.F.; Maldonado, G.; McCandless, L.C.; Greenland, S. Good practices for quantitative bias analysis. Int. J. Epidemiol. 2014, 43, 1969-1985. [CrossRef] [PubMed]

49. Xu, F.; Sternberg, M.R.; Markowitz, L.E. Men who have sex with men in the United States: Demographic and behavioral characteristics and prevalence of HIV and HSV-2 infection: Results from national health and nutrition examination survey 2001-2006. Sex. Transm. Dis. 2010, 37, 399-405. [CrossRef] [PubMed]

50. Kates, J.; Ranji, U.; Beamesderfer, A.; Salganicoff, A.; Dawson, L. Health and Access to Care and Coverage for Lesbian, Gay, Bisexual, and Transgender Individuals in the U.S.; Henry, J., Ed.; Kaiser Family Foundation: Menlo Park, CA, USA, 2014. 
51. Bird, J.D.; Voisin, D.R. "You're an open target to be abused": A qualitative study of stigma and HIV self-disclosure among black men who have sex with men. Am. J. Public Health 2013, 103, 2193-2199. [CrossRef] [PubMed]

52. Welles, S.L.; Corbin, T.J.; Rich, J.A.; Reed, E.; Raj, A. Intimate partner violence among men having sex with men, women, or both: Early-life sexual and physical abuse as antecedents. J. Community Health 2011, 36, 477-485. [CrossRef] [PubMed]

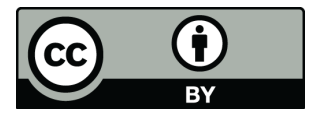

(C) 2018 by the authors. Licensee MDPI, Basel, Switzerland. This article is an open access article distributed under the terms and conditions of the Creative Commons Attribution (CC BY) license (http:/ / creativecommons.org/licenses/by/4.0/). 